\title{
Lattice Formation in Space for a Swarm of Pico Satellites
}

\author{
Carlo Pinciroli ${ }^{1}$, Mauro Birattari ${ }^{1}$, Elio Tuci ${ }^{1}$, Marco Dorigo ${ }^{1}$, \\ Marco del Rey Zapatero ${ }^{2}$, Tamas Vinko ${ }^{2}$, and Dario Izzo $^{2}$ \\ 1 IRIDIA, CoDE, Université Libre de Bruxelles, Brussels, Belgium \\ \{cpinciro, mbiro, etuci, mdorigo\}@ulb.ac.be \\ 2 European Space Agency, Noordwijk, The Netherlands \\ \{marco.del.rey.zapatero, tamas.vinko, dario.izzo\}@esa.int
}

\begin{abstract}
We present a distributed control strategy that lets a swarm of satellites autonomously form a lattice in orbit around a planet. The system, based on the artificial potential field approach, proposes a novel way to split the artificial field in two main terms: a global artificial field that gathers the satellites around a predefined meeting point, and a local term that allows a satellite to place itself in the correct position relative to its closest neighbors. We apply the method to the problem of forming a two dimensional hexagonal lattice, using the well-known Lennard-Jones potential as local artificial field. The control parameters have been obtained with a genetic algorithm to maximize the precision of the formed lattice. The precision does not depend on the number of satellites and convergence is achieved from all initial distributions of the satellites.
\end{abstract}

\section{Introduction}

In this paper, we propose a control system that allows a swarm of small spacecraft, called pico satellites, to build an hexagonal lattice in orbit around a planet. This is considered an important prerequisite for applications such as formation flight, coordinated observation [1,2], autonomous self-assembly of solar powered satellites [3], large antennas and large reflectors in space. The control system follows the principles of swarm intelligence: it is distributed and interactions among satellites are only local. Thanks to these characteristics, the system is highly scalable. Moreover, the system converges to the desired configuration for any initial distribution of the satellites. The validity of our results has been tested in simulations of up to 500 satellites. This paper builds on top of our preliminary work [4], in which we introduce the control system and we briefly study the precision of the lattice with control parameters chosen by hand in a flat space (no gravitational forces). In this paper, we optimize the control parameters for a real orbital environment and we study the precision of the formed lattice with a varying number of satellites and with different initial conditions.

The swarm is represented as a set of $N$ identical point-masses. Initially, the satellites are randomly distributed in space under the gravitational influence of a near planet. A point $\boldsymbol{p}$ orbiting around the planet is defined at design time as 


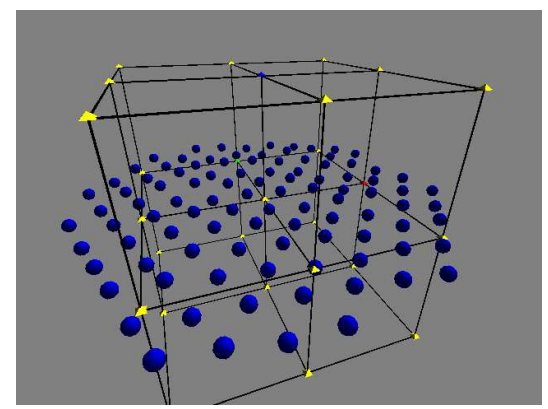

Fig. 1: An example of hexagonal lattice with 100 satellites.

\begin{tabular}{lrrr} 
Orbit & $\omega(\mathrm{rad} / \mathrm{s})$ & $R(\mathrm{~km})$ & $\mathrm{T}(\mathrm{s})$ \\
\hline \hline LEO & $1 \cdot 10^{-3}$ & 7,000 & 6,283 \\
GEO & $7.3 \cdot 10^{-5}$ & 42,000 & 86,071 \\
Amalthea & $1.5 \cdot 10^{-4}$ & 181,000 & 41,888 \\
Metis & $2.5 \cdot 10^{-4}$ & 129,000 & 25,133 \\
Io & $4.1 \cdot 10^{-5}$ & 421,600 & 153,248
\end{tabular}

Fig. 2: Different types of orbital environments considered. $\omega$ is the angular speed of rotation around the planet, $R$ is the distance from its center and $\mathrm{T}$ is the time needed to complete one orbit.

the origin of a reference frame. The control strategy we present in this paper lets the satellites position themselves around $\boldsymbol{p}$ to form a regular hexagonal lattice located on the $x y$ plane (see Figure 1). The satellites keep a mutual target distance $\sigma$ which is a control parameter fixed at design time. In our simulations, the motion of a satellite has been modeled with the Hill's system of differential equations [5] assuming that the orbit of $\boldsymbol{p}$ around the planet is circular. The satellites have a mass $m=100 \mathrm{~kg}$ and a thrusting capability $T_{\max }=50 \mathrm{mN}$. The swarm has been tested in various orbital scenarios, reported in Table 2: geostationary orbits (GEO), low Earth orbits (LEO), and Jupiter orbits close to those of its satellites Amalthea, Metis and Io.

The main goals of the work are to ensure that satellites are not lost in space, that collisions are avoided and that the overall system is scalable, i.e., that the control strategy does not depend on the number $N$ of satellites.

\section{The Control Strategy}

The control strategy studied in this work follows the artificial potential approach [6]. This idea has been first introduced for robot path planning [7] and proved effective also in satellite control problems [8]. The agent is imagined immersed in a virtual potential field calculated by its control system. The control action $\boldsymbol{u}$ is the virtual force due to the virtual potential. The goal position of the agent corresponds to the status of minimum energy in the potential field. The artificial potential approach has been applied for formation control of wheeled robots. Balch and Arkin [9] proposed a system in which a small group of robots, uniquely identified, keep preassigned relative positions with a very simple attraction potential. Spears et al. [10] devised a distributed system in which robots form an hexagonal lattice through local interactions inspired by gravitational forces. This work neglects collision avoidance and assumes the robots to be initially very tightly distributed.

The features of the task considered in this paper suggested a novel definition of the virtual potential field. The control strategy $\boldsymbol{u}$ has been expressed as the 


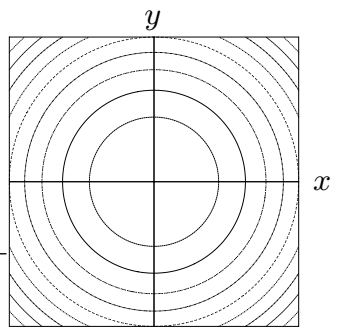

(a)

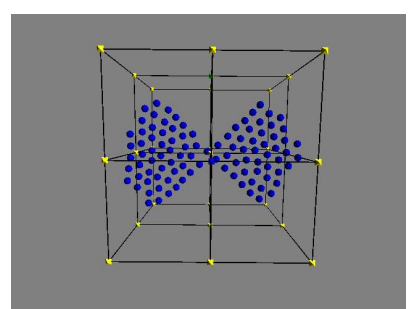

(b)

Fig. 3: (a) The equipotential curves of $g$ on the $x y$ plane (b) A butterfly shaped lattice obtained with a suitable $\boldsymbol{g}$ potential.

superposition of three contributions:

$$
\boldsymbol{u}=\boldsymbol{g}+\boldsymbol{l}+\boldsymbol{d}
$$

where $\boldsymbol{g}$ is a force that attracts each satellite towards the origin of the common reference frame, $\boldsymbol{l}$ is a force that creates locally flat lattices with the neighboring satellites while avoiding in-swarm collisions, and $\boldsymbol{d}$ is a damping factor analogous to viscosity, used to stabilize the behavior of the swarm and to ensure convergence.

\subsection{Global Attraction to the Origin}

We assume that the satellites know their position with respect to the reference frame defined by $\boldsymbol{p}$. This is not a stringent requirement in a space application because many well known techniques can be employed, spanning from the use of triangulation with fixed star positions to placing a special satellite in $\boldsymbol{p}$ that broadcasts its position in space.

Defining $\boldsymbol{q}$ as the position of a satellite with respect to $\boldsymbol{p}$, and defining the normalized vector $\overline{\boldsymbol{q}}=\left[\begin{array}{lll}\bar{q}_{x} & \bar{q}_{y} & \bar{q}_{z}\end{array}\right]^{T}=\boldsymbol{q} /\|\boldsymbol{q}\|$, then the virtual force that attracts satellites towards the origin is defined as:

$$
\boldsymbol{g}=-\eta\|\boldsymbol{q}\|^{2} \overline{\boldsymbol{q}}
$$

where $\eta$ is a design parameter. Thanks to virtual viscosity (term $\boldsymbol{d}$ of Equation 1 , see also Section 2.3), a satellite starting from any point in space converges, after some time, to the origin.

As shown in Figure 3a, sections of the potential that defines $\boldsymbol{g}$ cut parallel to the $x y$ plane are circle shaped. Therefore, the global shape of the swarm is a circle. Using a potential with a different section contour, it is possible to change the global shape of the formation. As an example, Figure $3 \mathrm{~b}$ depicts a butterfly shape obtained with a different $\boldsymbol{g}$. 


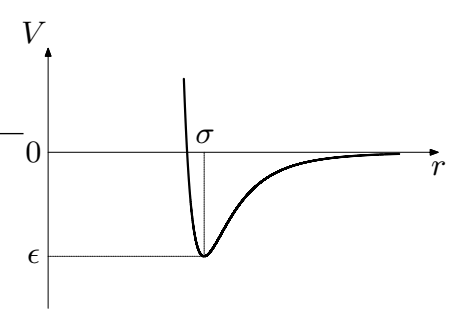

(a)

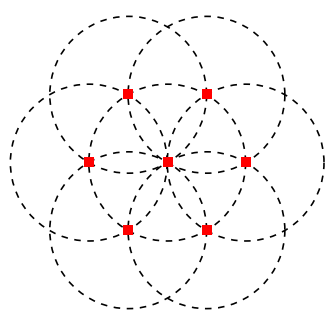

(b)

Fig. 4: (a) The Lennard-Jones potential (b) Its equilibrium state, an hexagonal lattice.

\subsection{Local Lattice Formation}

The local potential field lets a satellite interact with its neighbors to create a lattice, while avoiding collisions. In this work, the local potential is inspired by a simple and very well known model of molecular interaction, the Lennard-Jones potential [11]:

$$
V(r)=\epsilon\left[\left(\frac{\sigma}{r}\right)^{12}-2\left(\frac{\sigma}{r}\right)^{6}\right]
$$

$r$ being the distance between two molecules. The force $\boldsymbol{F}(r)$ between two molecules is given by

$$
\boldsymbol{F}(r)=-\nabla V(r)=-\frac{d}{d r} V(r) \hat{\boldsymbol{r}},
$$

where $\hat{\boldsymbol{r}}$ is a normalized vector directed as the line going from the center of the first molecule to the center of the second. This force is null when the distance coincides with the target distance $\sigma$; the force is increasingly repulsive as $r<\sigma$ decreases; the force is attractive when $r>\sigma$. As Figure 4a shows, the attraction is very strong when $r$ is not much larger than $\sigma$, but after a certain distance this force fades to zero, thus explaining the reason why we call this potential local. The stable arrangement of two molecules is such that they keep the mutual target distance $\sigma$ (in our experiments, $\sigma=300 \mathrm{~m}$ ). Increasing the number of molecules, the stable arrangement is an hexagon (see Figure $4 \mathrm{~b}$ ).

The design parameters of the potential are few and very intuitive to set: $\sigma$ is the mutual distance among the satellites in the lattice, while $\epsilon$ is the depth of the potential well, which accounts for the attractiveness and stability of the minimum located at distance $\sigma$. Notably, the lattice is formed on the basis of positional information only: no communication is needed.

From Equations 3 and 4, the magnitude of the virtual force parallel to the $x y$ plane between a satellite and its $i$-th neighbor is given by

$$
l_{i}^{x y}=-\frac{d}{d r} V(r)=\frac{12 \epsilon}{r}\left[\left(\frac{\sigma}{r}\right)^{12}-\left(\frac{\sigma}{r}\right)^{6}\right] .
$$

To flatten the lattice, a virtual force parallel to the $z$ axis is defined as $l_{i}^{z}=$ $-\psi \operatorname{sign}\left(r_{z}\right) r_{z}^{2}$, where $r_{z}$ is the projection of $\boldsymbol{r}=r \hat{\boldsymbol{r}}$ on the $z$ axis. The force 
is then $\boldsymbol{l}_{i}=\left[\begin{array}{lll}l_{i}^{x y} \bar{q}_{x} & l_{i}^{x y} \bar{q}_{y} & l_{i}^{z}\end{array}\right]^{T}$. Eventually, $\boldsymbol{l}$ is defined as the average of the virtual forces due to the $M$ closest neighbors (in our experiments, $M=6$ ):

$$
\boldsymbol{l}=\frac{1}{M} \sum_{i=1}^{M} \boldsymbol{l}_{i}
$$

It is possible to control the shape of the local lattice by using a different potential. A natural choice is a molecular model already studied in crystallography. In other terms, the work presented here suggests a link between crystallography and lattice formation in robotics.

\section{$2.3 \quad$ Ensuring Convergence}

The virtual forces $\boldsymbol{g}$ and $\boldsymbol{l}$ are defined by conservative fields. This means that convergence is impossible without a dissipative term. To obtain convergence, we imagine that the satellites are immersed in a viscous medium. Thus, the expression of $\boldsymbol{d}$ is analogous to viscosity: $\boldsymbol{d}=-\xi \dot{\boldsymbol{q}}$, where $\xi$ is a design parameter, usually smaller than 0.2 .

\subsection{Formation Stabilization After Convergence}

Experiments revealed that once the swarm converges to its final configuration, the satellites oscillate around their equilibrium points, thus wasting propellant. A solution to this problem is increasing the damping factor $\xi$ after the final configuration has been reached:

$$
\dot{\xi}= \begin{cases}\xi_{\text {conv }} e^{-\xi / 2} & \text { if } \xi<\xi_{\text {stab }}, \\ 0 & \text { otherwise. }\end{cases}
$$

The value of $\xi_{\text {conv }}$ is the one that ensures convergence when satellites form the lattice (see Section 2.3). The value $\xi_{s t a b}$ for which oscillations disappear depends on the orbit at which $\boldsymbol{p}$ is located. In our experiments $\xi_{\text {stab }}=0.7$.

A further problem is when to trigger the stabilization. Currently we adopt a simple time-based criteria. Each satellite individually measures the time elapsed since the beginning of the shape formation process. After a certain time, stabilization is triggered. A more elegant method would be to trigger the stabilization with a distributed consensus algorithm [12].

\section{Results}

Experimental evaluation shows that even with suboptimal parameters the system works reasonably well, although good parameters for an orbital environment are not equally good for another [4]. Here, we optimize the parameters to minimize positioning errors in the lattice. With these parameters, we study scalability. Finally, we test the dependence of the control system on initial conditions (placement of satellites). 


\begin{tabular}{lr} 
Parameter & Value \\
\hline \hline Number of generations & 1000 \\
Population size & 50 \\
Mutation probability & 0.2 \\
Crossover probability & 0.9 \\
Elitism & the best survives
\end{tabular}

Fig. 5: Parameters of the genetic algorithm employed for setting the control parameters.

\begin{tabular}{lr} 
Parameter & Value \\
\hline \hline$\eta$ & $1.6295 \cdot 10^{8}$ \\
$\psi$ & $5.96201 \cdot 10^{8}$ \\
$\epsilon$ & $4.5332 \cdot 10^{4}$ \\
$\xi$ & 0.165984
\end{tabular}

Fig. 6: Values of the control parameters obtained via the genetic algorithm.

\subsection{Optimizing the Control Parameters}

Good values of some control parameters, such as $\eta, \psi, \epsilon$ and $\xi$, are not easy to find. We chose to optimize them with Goldberg's simple genetic algorithm [13]. Table 5 summarizes the parameter values used for the genetic algorithm.

Evolutions were performed with 10 satellites in a GEO environment. The trials lasted 1000 time steps, each time step being $12.5 \mathrm{~s}$ long. The placement of a satellite has been evaluated as follows:

$$
\chi_{i}=\frac{1}{N_{i}} \sum_{j \in \mathcal{N}_{i}} \frac{\left|\sigma-r_{i j}\right|}{\sigma}
$$

where $\mathcal{N}_{i}$ is the set containing the $N_{i}$ closest neighbors of satellite $i$ and $r_{i j}$ is the relative distance between the satellites $i$ and $j$ at the final lattice acquisition time. The genetic algorithm minimizes the worst satellite placement, defined as $\chi=\max _{i} \chi_{i}$. The best control parameters that we obtained are reported in Table 6 . They yield a score $\chi=0.012842$, which corresponds to a positioning error of $3.85 \mathrm{~m}(\sigma=300 \mathrm{~m})$.

\section{$3.2 \quad$ Scalability}

Scalability makes it possible to optimize the parameters with a minimal number of satellites, thus finding quickly a convenient setup. Figure 7 reports the behavior of the placement error for different numbers of satellites. The placement error is calculated as $\bar{\chi}=\frac{1}{N} \sum_{i=1}^{N} \chi_{i}$. Although the parameters were obtained through trials involving only 10 satellites, $\bar{\chi}$ remains practically constant around the value 0.02 (that corresponds to $6 \mathrm{~m}$ ), with a minimum of $0.007(2.1 \mathrm{~m})$ and a maximum of $0.035(10.5 \mathrm{~m})$. Only with 500 satellites the maximum error is slightly larger: $0.088(26.4 \mathrm{~m})$.

\subsection{Initial Conditions}

Convergence to the final structure can be mathematically proven by the presence of the global attractor located at the origin of the virtual global field and by the known results about the Lennard-Jones potential. 


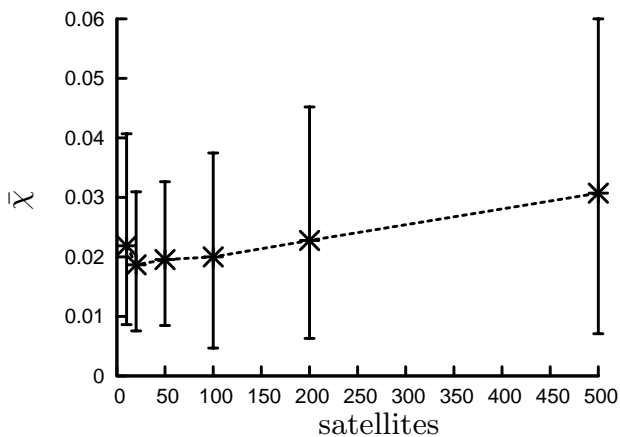

Fig. 7: Average placement error for a different number of satellites. \begin{tabular}{lr} 
Distribution & $\bar{\chi}$ \\
\hline \hline Centered Cubic & 0.0215913 \\
Centered Spheric & 0.0199159 \\
Shifted Cubic & 0.0207984 \\
Shifted Spheric & 0.0191019
\end{tabular}

Fig. 8: Placement error $\bar{\chi}$ obtained with different initial spatial distributions.

Table 8 shows the results of a set of experiments run to test if $\bar{\chi}$ is affected by the initial spatial distribution of the swarm. In the centered cubic distribution, the satellites are placed uniformly in a cube with side of $6 \mathrm{~km}$ and centered around the origin. The centered spheric distribution is a hollow sphere centered around the origin with radius $3 \mathrm{~km}$ and $300 \mathrm{~m}$ thick. The shifted distributions are centered in point $\left[\begin{array}{lll}3 & 3 & 3\end{array}\right]$ (coordinates in $\mathrm{km}$ ). For all the experiments, the same experimental conditions described in Section 3.1 have been used with swarms of 100 satellites. The results show that $\bar{\chi}$ has values similar to those found for the scalability tests.

\section{Conclusions}

The presented work deals with a decentralized control strategy for swarms of satellites that allows them to autonomously form a bi-dimensional hexagonal lattice under the gravitational influence of a near planet. The method is based on the artificial potential field approach. In the paper, a novel way of defining the potential is proposed. This method allows the designer to split the problem of forming the lattice into two more intuitive subproblems: an artificial field attracts globally the satellites towards a meeting point and controls the shape of the formation; another artificial field takes care of defining the interactions among the satellites to form local lattices. In this work, the Lennard-Jones potential has been used to define the local field. The control parameters to be set by the designer are few and very intuitive, and acceptable results can be obtained even by setting the parameters by hand. We have optimized the control parameters to minimize the placement error and results show that such error is independent of the number of satellites and of the initial spatial distribution of the swarm.

The way here proposed to define the artificial potential field suggests a possible link between lattice formation in robotics and known results in crystallography. We plan to further study this link by trying other potentials that are known in the literature. 
Acknowledgments. This research was funded by the European Space Agency under the Ariadna scheme. It was also partially supported by COMP2SYS and by the ANTS project. COMP2SYS is a Marie Curie Early Stage Training Site funded by the European Commission under contract MEST-CT-2004-505079. ANTS is an Action de Recherche Concertée funded by the French Community of Belgium. Mauro Birattari and Marco Dorigo acknowledge support from the Belgian F.R.S.-FNRS.

\section{References}

1. Curtis, S., Mica, J., Nuth, J., Marr, G., Rilee, M., Bhat, M.: ANTS (Autonomous Nano-Technology Swarm): An artificial intelligence approach to asteroid belt resource exploration, International Astronautical Federation, 51th Congress (2000)

2. D'Arrigo, P., Santandrea, S.: The APIES mission. ASTRIUM Ltd./ESA-ESTEC Feasibility Study A0/1-3846/02/NL/JA, Stevenage, UK (2004)

3. Mori, M., Nagayama, H., Saito, Y., Matsumoto, H.: Summary of studies on space solar power systems of the national space development agency of Japan. Acta Astronautica 54(5) (2004) 337-345

4. Pinciroli, C., Birattari, M., Tuci, E., Dorigo, M., del Rey Zapatero, M., Vinko, T., Izzo, D.: Self-organizing and scalable shape formation for a swarm of pico satellites. In: Proceedings of the NASA/ESA Conference on Adaptive Hardware and Systems (AHS-2008), Washington, DC, IEEE-CS Press (2008) in press.

5. Clohessey, W., Wiltshire, R.: Terminal guidance systems for satellite rendez-vous. Journal of the Aerospace Sciences 27(9) (1960) 653-658

6. Khatib, O.: Real-time obstacle avoidance for manipulators and mobile robots. International Journal of Robotics Research 5(1) (1986) 90-98

7. Ge, S.S., Cui, Y.I.: Dynamic motion planning for mobile robots using potential field method. Autonomous Robots 13(3) (2002) 207-222

8. Badawy, A., McInnes, C.: On-orbit assembly using superquadric potential fields. Journal of Guidance, Control, and Dynamics 31(1) (2008) 30-43

9. Balch, T., Arkin, R.C.: Motor schema-based formation control for multiagent robot teams. In Lesser, V., Gasser, L., eds.: Proceedings of the First International Conference on Multiagent Systems (ICMAS'95), San Francisco, CA, AAAI Press (1995) 10-16

10. Spears, W., Spears, D., Hamann, J., Heil, R.: Distributed, physics-based control of swarms of vehicles. Autonomous Robots 17(2-3) (2004)

11. Kittel, C.: Introduction to Solid State Physics. Wiley, New York (1986)

12. Lamport, L.: Lower bounds on asynchronous consensus. In Schiper, A., Shvartsman, A.A., Weatherspoon, H., Zhao, B.Y., eds.: Future Directions in Distributed Computing. Volume 2584 of Lecture Notes in Computer Science., Berlin, Springer (2003) 22-23

13. Goldberg, D.E.: Genetic Algorithms in Search, Optimization and Machine Learning. Addison-Wesley, Boston, MA (1989) 